\title{
Prognostic value of log odds of positive lymph nodes in node-positive lung squamous cell carcinoma patients after surgery: a SEER population-based study
}

\author{
Yue Yu ${ }^{1 \#}$, Peng Zhang ${ }^{1 \#}$, Renqi Yao ${ }^{2,3 \#}$, Junnan Wang ${ }^{1,4}$, Pei Wang ${ }^{1}$, Xiaofei Xue ${ }^{1}$, Jian Xiao ${ }^{1}$, Zhinong Wang ${ }^{1}$ \\ ${ }^{1}$ Department of Cardiothoracic Surgery, Changzheng Hospital, Naval Medical University, Shanghai, China; ${ }^{2}$ Trauma Research Center, Fourth \\ Medical Center of the Chinese PLA General Hospital, Beijing, China; ${ }^{3}$ Department of Burn Surgery, Changhai Hospital, Naval Medical University, \\ Shanghai, China; ${ }^{4}$ Medical Research Center of War Injuries and Trauma, Changzheng Hospital, Naval Medical University, Shanghai, China \\ Contributions: (I) Conception and design: J Xiao, Z Wang; (II) Administrative support: X Xue, Z Wang; (III) Provision of study materials or patients: \\ None; (IV) Collection and assembly of data: Y Yu, P Zhang, J Wang; (V) Data analysis and interpretation: Y Yu, R Yao, P Wang; (VI) Manuscript \\ writing: All authors; (VII) Final approval of manuscript: All authors. \\ \#These authors contributed equally to this work. \\ Correspondence to: Jian Xiao, MD, PhD; Zhinong Wang, MD, PhD. Department of Cardiothoracic Surgery, Changzheng Hospital, Naval Medical \\ University, No. 415 Fengyang Road, Huangpu District, Shanghai 200040, China. Email: 18916066266@189.cn; wangzn007@smmu.edu.cn.
}

Background: Log odds of positive lymph nodes (LODDS) is a novel and promising ratio-based lymph node (LN) staging system in many malignancies. This study aimed to evaluate the prognostic value of LODDS, and comprehensively compare the prognostic predictive performance of LODDS with the American Joint Committee on Cancer (AJCC) N classification, number of positive lymph node (NPLN), and lymph node ratio (LNR) among node-positive lung squamous cell carcinoma (SCC) patients after surgery.

Methods: We identified 2,561 patients with N1/N2 stage SCC diagnosed between 2004 and 2014 from the Surveillance, Epidemiology, and End Results (SEER) database. X-tile analysis was used to calculate the optimal cut-off value for each staging system. Univariable and Multivariable Cox regression analyses were used to assess the association of cancer-specific survival (CSS), and overall survival (OS) with N, NPLN, LNR, and LODDS, separately, and integrally. Moreover, linear trend $\chi^{2}$ score, likelihood ratio (LR) test, Akaike information criterion (AIC), and Harrell concordance index (C-index) were adopted as criteria for assessing the predictive ability of each model.

Results: The optimal cut-off values for NPLN, LNR, and LODDS were 3, 0.28, and -0.37, respectively. N, NPLN, LNR, and LODDS were identified as independent prognostic predictors for CSS and OS in patients with SCC when each of them was incorporated into multivariable Cox model separately. Additionally, LODDS had the higher linear trend $\chi^{2}$ score, higher LR $\chi^{2}$ test score, lower AIC, and higher C-index compared to the other three systems. Moreover, a combination of N, NPLN, and LODDS was superior to any staging system alone for predicting prognosis.

Conclusions: LODDS showed better predictive performance than N, NPLN, and LNR among patients with node-positive SCC after surgery. A combination of LODDS and the current AJCC TNM classification has the potential for becoming a better staging method to more precisely predicting prognosis.

Keywords: Log odds of positive lymph nodes (LODDS); non-small cell lung cancer (NSCLC); prognosis; squamous cell carcinoma (SCC); Surveillance, Epidemiology, and End Results (SEER)

Submitted Feb 02, 2020. Accepted for publication Jun 30, 2020.

doi: $10.21037 /$ tlcr-20-193

View this article at: http://dx.doi.org/10.21037/tlcr-20-193 


\section{Introduction}

Lung cancer has been the leading cause of cancer-related deaths worldwide. Approximately 1.8 million people are diagnosed with lung cancer, and 1.6 million people die as a result of the cancer each year (1-3). Lung squamous cell carcinoma (SCC) is a unique clinical and the second largest histological subtype of non-small cell lung cancer (NSCLC) that accounts for about $30-40 \%$ of all lung cancers $(4,5)$. Compared to lung adenocarcinoma (AC), SCC is associated with distinct epidemiological features, lack of effective targeted treatment options, and poor clinical prognosis $(3,6,7)$. This underscores the importance of an accurate staging system that can precisely predict the prognosis of SCC, and help implementing appropriate treatment.

Lymph node (LN) status is considered as a major determinant of prognosis, and a combination of anatomic location of involved LNs and the total number of dissected lymph nodes (NDLN) has been applied in the staging of various malignant tumors for better therapeutic strategies (8-12). Prognosis for NSCLC is mainly dependent on the latest 8th American Joint Committee on Cancer (AJCC) Tumor Node Metastasis (TNM) staging system, which recommends station-based LN sampling/dissection and tumor staging $(13,14)$. However, this currently used staging system does not take into any number- or ratiobased nodal system classification, which might influence the precision of prognosis evaluation, the extent of dissection of LNs in surgery, and postoperative treatment for patients with NSCLC $(15,16)$. Several previous studies have demonstrated that higher values of number of positive lymph node (NPLN), and lymph node ratio (LNR) were associated with worse survival outcomes for NSCLC (17-21).

Log odds of positive lymph nodes (LODDS) is a novel ratio-based nodal staging system, defined as the logarithm of the ratio between NPLN and the total number of negative LNs harvested. LODDS is currently being used to as a more effective prognostic predictor in several types of malignant tumors (16,22-31). However, until now, only a few studies have attempted exploring the prognostic validation of LODDS in NSCLC or lung AC alone (16,25-27), and no specified research so far has focused on its prognostic role in SCC, the second most common histological subtype of NSCLC. Moreover, whether LODDS was superior to other systems and could be used to optimize the current nodal classification is still unclear.
Based on the Surveillance, Epidemiology, and End Results (SEER) database, our first aim was to evaluate the prognostic role of LODDS staging system for predicting long-term prognosis of postoperative node-positive lung SCC patients. In addition, our second aim was to comprehensively compare the prognostic performance of the AJCC N classification, NPLN, LNR, and LODDS systems and to identify a superior staging system for predicting survival outcomes of patients with SCC. We present the following article in accordance with the TRIPOD reporting checklist (available at http://dx.doi. org/10.21037/tlcr-20-193).

\section{Methods}

\section{Data source}

The data from this study were collected from the SEER 18 registries research database of the National Cancer Institute (NCI). SEER database includes data of approximately $34.6 \%$ of the population of the United States (32). We obtained permission to access the dataset (authorization code: 11874-Nov2018). The data were extracted from the SEER database via SEER*Stat software (version 8.4.6; http://seer. cancer.gov/seerstat/). This study utilized the anonymous data available in the SEER database, and hence the requirement for informed consent was waived. This study was complied with the Declaration of Helsinki (as revised in 2013), and approved by the committee of Institutional Review Board of Changzheng Hospital (Naval Medical University, Shanghai, China). The content is solely the responsibility of the authors and does not necessarily represent the official views of the SEER database and NCI.

\section{Population selection}

From the SEER database, we identified patients, histologically diagnosed with the primary lung SCC (histological codes: 8052/3, 8070/3, 8071/3, 8072/3, $8073 / 3,8074 / 3,8083 / 3$, and $8084 / 3$ ) based on the $3 \mathrm{rd}$ edition of the International Classification of DiseaseOncology (ICD-O-3) His/Behave, malignant. The study period was chosen from 2004 to 2014 to guarantee an adequate duration of follow-up time. Patients who underwent a radical surgical procedure and systematic $\mathrm{LN}$ dissection, with evidence of N1/N2 stage disease were selected into the study cohort. Patients were excluded 
who (I) aged $<18$ years; (II) were identified by autopsy or a death certificate only; (III) received a diagnosis while in a nursing home or hospice; (IV) had diagnosis of any other cancer; (V) were at N0/N3 stage disease; (VI) had distant metastasis (M1); (VII) had a history of preoperative radiotherapy; (VIII) did not undergo radical surgery or systematic lymphadenectomy; (IX) underwent pathological examination of resected specimens or invasive mediastinal staging prior to surgery; $(\mathrm{X})$ survived less than one month after surgery; (XI) had missing data related to TNM staging system, NDLN, NPLN, and survival outcomes. The TNM stages of the patients in the SEER database were updated in line with the 8th edition of the AJCC criteria (33).

\section{Study covariates}

From the SEER database, the baseline demographics data of patients including age at diagnosis, sex, race, Contract Health Service Delivery Areas (CHSDA) region, marital status, insurance status, and cost-of-living index were extracted. We also included data on baseline tumor characteristics that included year of diagnosis, primary site, laterality, tumor differentiation, $\mathrm{T}$ stage, and $\mathrm{N}$ stage. In addition, treatment strategies including surgical intervention, radiotherapy, chemotherapy, NDLN, and NPLN were extracted from the database.

\section{Identification of cut-off values for NDLN, LPLN, LNR, and LODDS systems}

LODDS was calculated using the formula: $\log (\mathrm{NPLN}+0.50) /(\mathrm{NDLN}-\mathrm{NPLN}+0.50)$, in which 0.50 was added to both the numerator and denominator to avoid an infinite number (34). LNR was defined as the ratio of positive LNs divided by the total number of dissected LNs and calculated using the formula: NPLN/NDLN. Four continuous variables (NDLN, NPLN, LNR, and LODDS) were all dichotomized via the $\mathrm{X}$-tile software (version 3.6.1; Yale University, New Haven, CT, USA) based on the maximal log-rank chi-square value, which represented the greatest group difference in outcome probability (20,35-38). In our study, NDLN was divided into NDLN1 (range, 1 to 10) and NDLN2 (range, 11 to 82), and NPLN was grouped into NPLN1 (range, 1 to 3 ) and NPLN2 (range, 4 to 33). In addition, LNR was categorized into LNR1 (range, 0.01 to 0.28 ) and LNR2 (range, 0.29 to 1.00 ), and LODDS was classified into LODDS1 (range, -1.70 to -0.37 ) and LODDS2 (range, -0.36 to 1.40 ).

\section{Outcomes}

In the current study, lung cancer-specific survival (CSS) and overall survival (OS) were chosen as primary endpoints of our study. Causes of death were coded by the SEER database according to information extracted from the death certificate data. CSS was defined as the time from diagnosis to SCC-related death, while OS referred to the time from diagnosis to all-cause death. The SEER program is updated annually, including patients' information on follow-up and prognosis. In this study, the latest patient information was released in December 2016. Therefore, the survival time was measured as the number of months from diagnosis until death or the last follow-up (December 31, 2016) for censored observations.

\section{Statistical analysis}

In the current study, continuous variables were presented with the mean [standard deviation (SD)] or the median [interquartile range (IQR)], while ranked or categorical variables were displayed as count and percentages. Baseline characteristics of study cohort stratified by LODDS staging system were summarized and compared by using Student's $t$-test, Kruskal Wallis rank test, Pearson's $\chi^{2}$ test or Fisher's exact test as appropriated.

Kaplan-Meier (KM) analysis was used to calculate 1-, $3-$, and 5-year CSS and OSS rate, and log-rank test was used to compare differences between groups. Moreover, a 3-step Cox proportional hazards regression analysis was performed to determine the association between different LN staging systems and prognosis, with the hazard ratio (HR) and a 95\% confidence interval (CI) reported. Firstly, univariable analysis was conducted to identify which among those confounders were potential prognostic factors. Variables which were of statistical significance $(\mathrm{P}<0.100)$ in the univariable Cox regression model were subsequently incorporated into the multivariable Cox regression analysis. Secondly, we separately included the N (Model 1), NPLN (Model 2), LNR (Model 3), and LODDS (Model 4) in four different multivariable Cox regression models. Thirdly, all these four staging systems and other potential predictors in the univariable analysis were simultaneously entered into the Cox regression model (Model 5). Performance of the predictive models was evaluated from multiple dimensions, including monotonicity, homogeneity, and discrimination. The linear trend $\chi^{2}$ score were used to assess discriminatory ability and monotonicity of each model. The likelihood-ratio 
(LR) $\chi^{2}$ test was used to assess homogeneity between groups. Akaike Information Criterion (AIC) was applied to test goodness of fit $(39,40)$. Harrell concordance index (C-index) was calculated to assess the accuracy of prediction (41). Correlations between the N, NPLN, LNR, and LODDS were visualized by scatter plots and evaluated by Pearson correlation coefficient (r). Besides, sensitivity analysis was performed accordingly.

For all statistical analyses, a two-sided $\mathrm{P}<0.050$ was considered to be statistical significance. All statistical analyses were performed using SPSS software (version 22.0; IBM Corporation, St. Louis, Missouri, USA) and R software (version.3.6.1; The R Project for Statistical Computing, TX, USA; http://www.r-project.org). This study was approved by the Ethics Committee of Changzheng Hospital
(No. ChiCTR-IOR-19005542).

\section{Results}

\section{Patients characteristics}

Between January 1, 2004 and December 31, 2014, the SEER database collected data on 109,348 patients diagnosed with malignant SCC. After applying the inclusion and exclusion criteria, the final study cohort consisted of 2,561 SCC patients. The selection process of our study population is summarized in Table S1. Baseline demographic and clinicopathological characteristics are presented stratified by the LODDS system in Table 1. Among 2,561 enrolled patients, there were $1,760(68.72 \%)$ patients diagnosed

Table 1 Baseline characteristics of study population stratified by the LODDS system

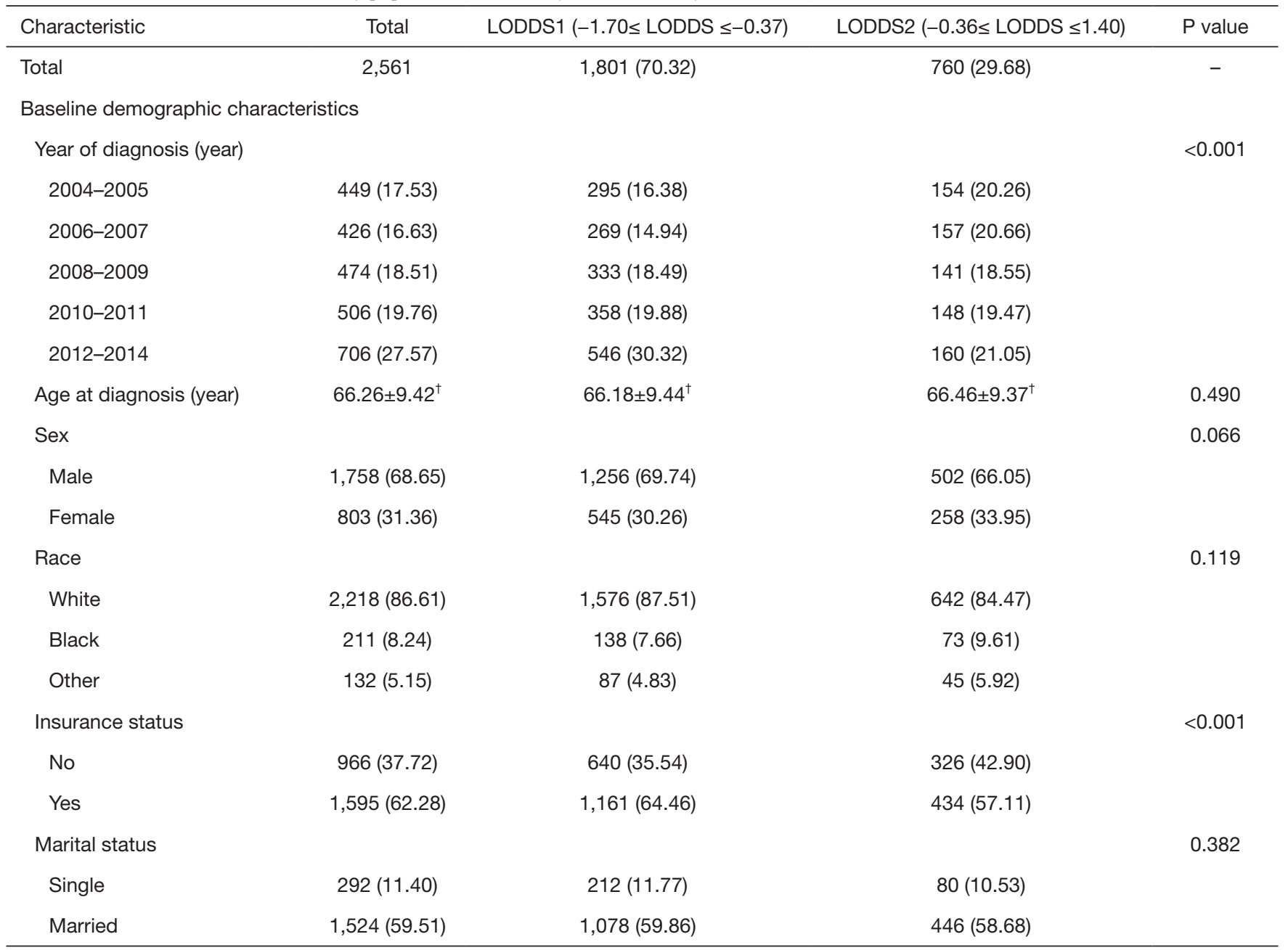

Table 1 (continued) 
Table 1 (continued)

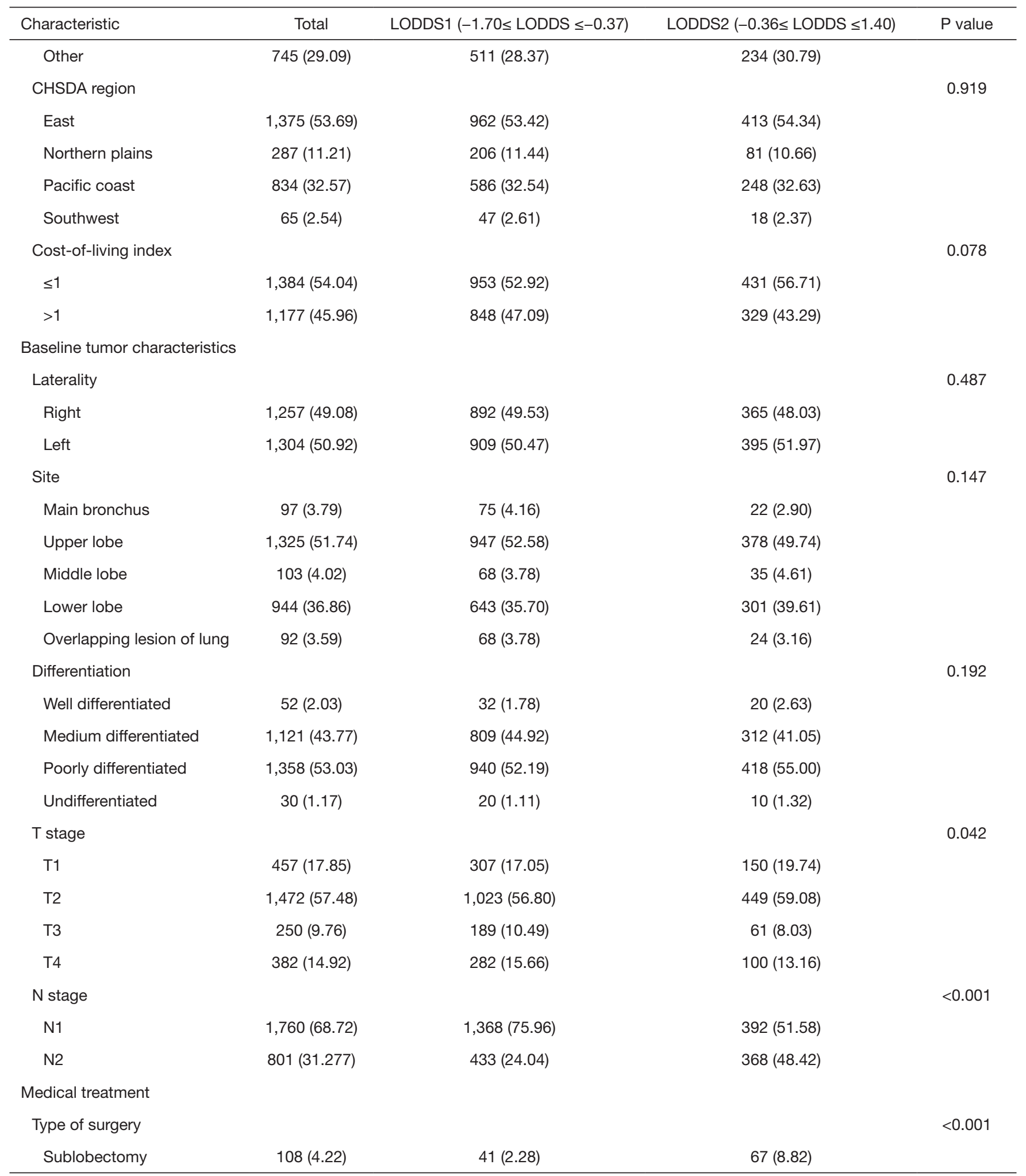

Table 1 (continued) 
Table 1 (continued)

\begin{tabular}{|c|c|c|c|c|}
\hline Characteristic & Total & LODDS1 $(-1.70 \leq$ LODDS $\leq-0.37)$ & LODDS2 $(-0.36 \leq$ LODDS $\leq 1.40)$ & $P$ value \\
\hline Pneumonectomy & $182(7.11)$ & $132(7.33)$ & $50(6.58)$ & \\
\hline Unspecific & $622(24.29)$ & $455(25.26)$ & $167(21.97)$ & \\
\hline Radiation & & & & $<0.001$ \\
\hline Yes & $535(20.89)$ & $303(16.82)$ & $232(30.53)$ & \\
\hline Chemotherapy & & & & 0.056 \\
\hline None/unknown & $1,071(41.82)$ & $775(43.03)$ & $296(38.95)$ & \\
\hline Yes & $1,490(58.18)$ & $1,026(56.97)$ & $464(61.05)$ & \\
\hline NPLN & & & & $<0.001$ \\
\hline NPLN1 [1-3] & $2,057(80.32)$ & $1,617(89.78)$ & $440(57.90)$ & \\
\hline NPLN2 [4-33] & $504(19.68)$ & $184(10.22)$ & $320(42.11)$ & \\
\hline LNR & & & & $<0.001$ \\
\hline LNR1 (0.01-0.28) & $1,785(69.70)$ & $1,785(99.11)$ & $0(0.00)$ & \\
\hline LNR2 (0.29-1.00) & $776(30.30)$ & $16(0.89)$ & $760(100.00)$ & \\
\hline
\end{tabular}

Note: data were presented as numbers with percentage of study population in brackets. ${ }^{\dagger}$, variable (age at diagnosis) was presented with the mean \pm standard deviation (SD). LODDS, log odds of positive lymph nodes; CHSDA, contract health service delivery areas; NDLN, number of dissected lymph nodes; NPLN, number of positive lymph nodes; LNR, lymph node ratio.

with N1 stage disease and 801 (31.28\%) patients diagnosed with $\mathrm{N} 2$ stage disease, with a mean age of $66.26 \pm 9.42$ years. Moreover, the mean and median NDLN were $13.58 \pm 9.61$ and 11.00 (IQR, 4.00 to 18.00), and the mean and median NPLN were $2.52 \pm 2.42$ and 2.00 (IQR, 1.00 to 3.00). In addition, the mean and median LNR were $0.25 \pm 0.22$ and 0.180 (IQR, -0.85 to 0.33 ), and the mean and median LODDS were $-0.52 \pm 0.45$ and -0.560 (IQR, 0.10 to -0.28 ). For the LODDS system, 1,801 (70.32\%) patients were in the LODDS1 group, and 760 (29.68\%) patients were in the LODDS2 group. Compared with the LODDS1 group, the patients included in the LODDS2 group were more likely to receive sublobectomy $(\mathrm{P}<0.001)$, and postoperative radiotherapy $(\mathrm{P}<0.001)$ with a higher rate of $\mathrm{N} 2$ stage, less NDLN $(\mathrm{P}<0.001)$, more NPLN $(\mathrm{P}<0.001)$, and a higher value of $\mathrm{LNR}(\mathrm{P}<0.001)$. In addition, the $\mathrm{T}$ stage distribution was more advanced in the LODDS1 group $(\mathrm{P}=0.042)$.

\section{Survival analysis}

The median follow-up time for the entire cohort was 52 months (IQR, 33-81 months). A total of 1,564 (61.07\%) patients died from any cause, and 1,212 (47.33\%) patients died from SCC at the end of the study period (December 2016). The cumulative 1-, 3- and 5-year CSS rates for LODDS1 patients were $84.4 \%$ (95\% CI, 82.7-86.1\%), $61.1 \%(95 \% \mathrm{CI}, 58.7-63.6 \%)$, and $52.7 \%(95 \% \mathrm{CI}$, 50.1-55.4\%) respectively, and were $75.7 \%$ (95\% CI, $72.7-78.9 \%$ ), $43.2 \%$ (95\% CI, 39.5-47.1\%), and $33.7 \%$ (95\% CI, 30.0-37.7\%) for LODDS2 patients, respectively (Tables S2,S3). As shown in Figures 1,2, patients with N2 stage, more positive LNs dissected, or higher values of LNR and LODDS were significantly correlated with worse CSS and OS (all log-rank $\mathrm{P}<0.001$ ). Univariable Cox regression model suggested that age at diagnosis, sex, insurance status, laterality, $\mathrm{N}$ stage, T stage, radiotherapy, 
A
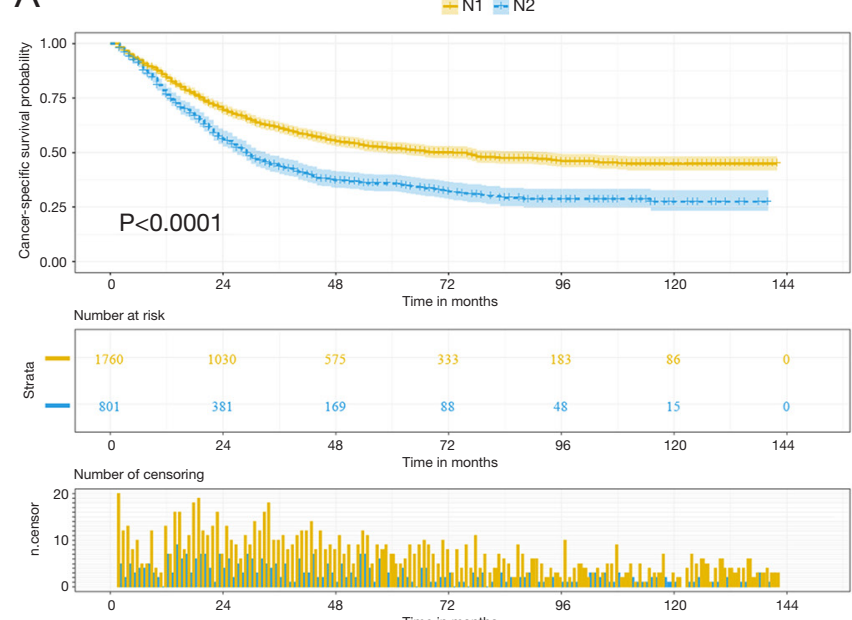

C
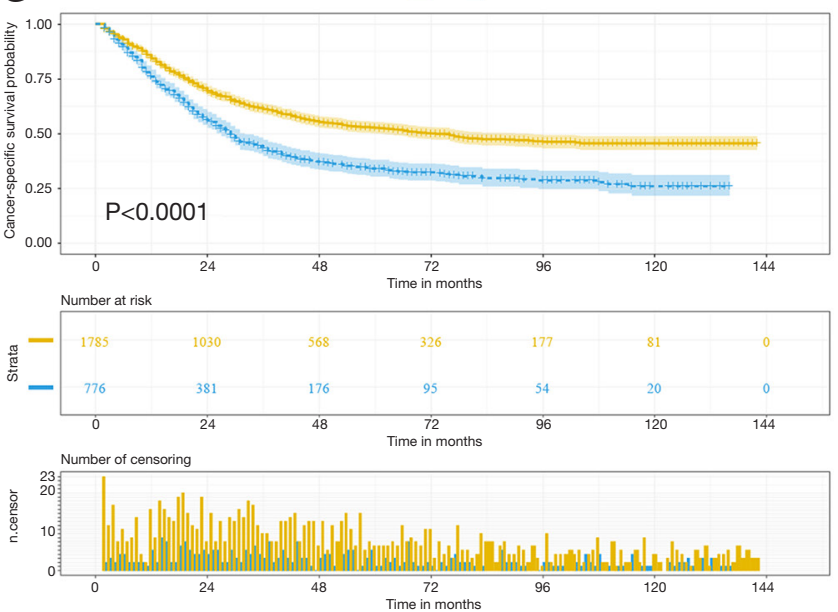

B
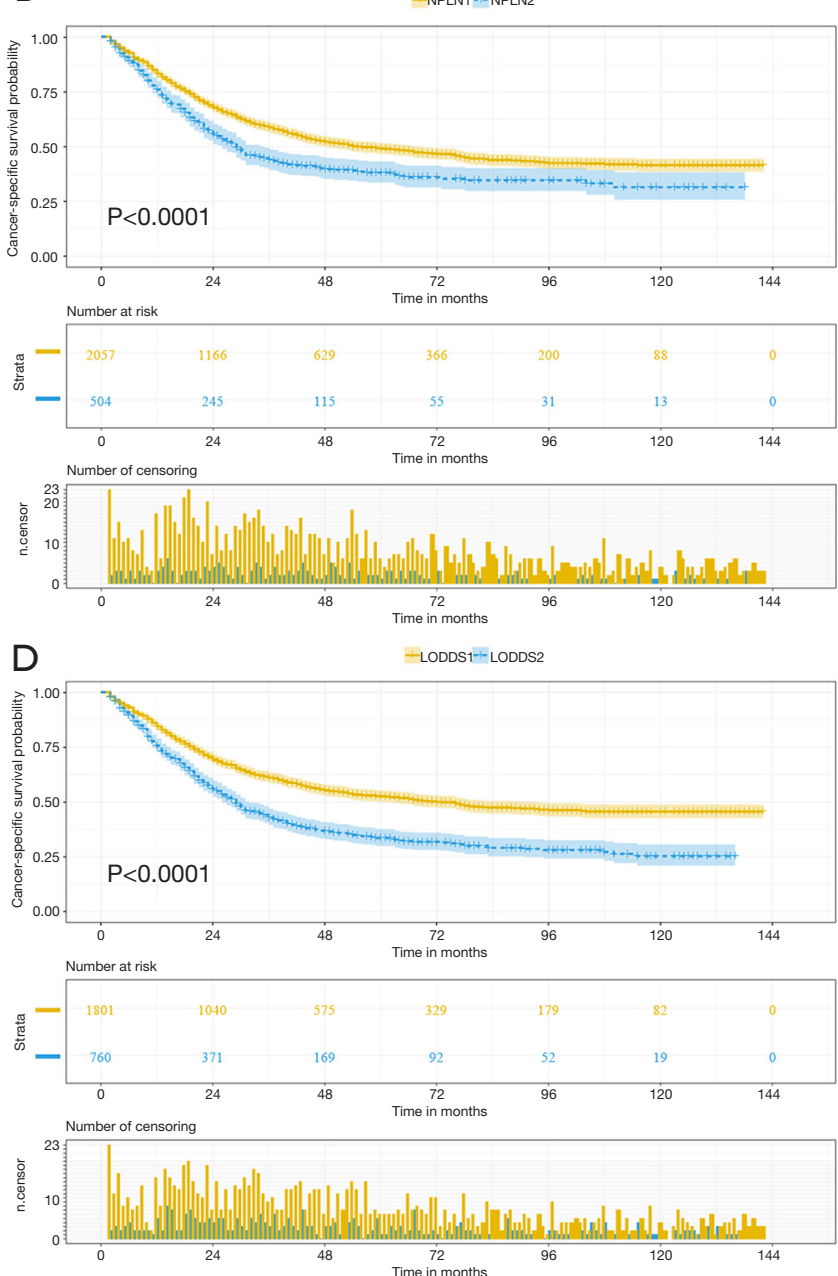

Figure 1 Kaplan-Meier estimates of cancer-specific survival for patients with N1 /N2 stage SCC after surgery according to (A) N, (B) NPLN, (C) LNR, and (D) LODDS staging systems. SCC, squamous cell carcinoma; NPLN, number of positive lymph nodes; LNR, lymph node ratio; LODDS, log odds of positive lymph nodes.

chemotherapy, NDLN, NPLN, LNR, and LODDS were potential prognostic factors for CSS in Table 2 (all $\mathrm{P}<0.100$ ). Likewise, the potential predictors for OS were also reported in Table 2. Based on the results in the univariable analysis, the N (Model 1), NPLN (Model 2), LNR (Model 3), and LODDS (Model 4) were separately incorporated into four different Cox regression models combined with the same confounders. Multivariable Cox regression analysis (Model 1-4) demonstrated that all the four staging systems $(\mathrm{N}, \mathrm{NPLN}, \mathrm{LNR}$, and LODDS) were all independent prognostic factors for CSS and OSS in Tables S4,S5. The respective HRs and 95\% CIs were as follows. For N, HR $=1.632(95 \%$ CI, $1.445-1.843)$ for CSS, and HR $=1.519$
(95\% CI, 1.363-1.692) for OS; for NPLN, HR $=1.588$ (95\% CI, 1.380-1.827) for CSS, and HR $=1.491$ (95\% CI, 1.313-1.692) for OS; for LNR, HR =1.648 (95\% CI, 1.464 1.856) for CSS, and HR $=1.511$ (95\% CI, 1.359-1.681) for OS; for LODDS, HR $=1.661$ (95\% CI, 1.475-1.870) for CSS, and HR $=1.525$ (95\% CI, 1.371-1.697) for OS. Then, all the four staging systems and significant factors in the univariable analysis were incorporated integrally into one Cox regression model (Model 5). The results showed that $\mathrm{N}$ (CSS: HR $=1.482 ; 95 \%$ CI, 1.307-1.681; OS: HR =1.403; 95\% CI, 1.255-1.569), NPLN (CSS: HR $=1.214 ; 95 \%$ CI, 1.031-1.429; OS: $\mathrm{HR}=1.193 ; 95 \% \mathrm{CI}, 1.030-1.381$ ), and LODDS (CSS: HR $=1.373 ; 95 \%$ CI, 1.186-1.591; OS: 
A
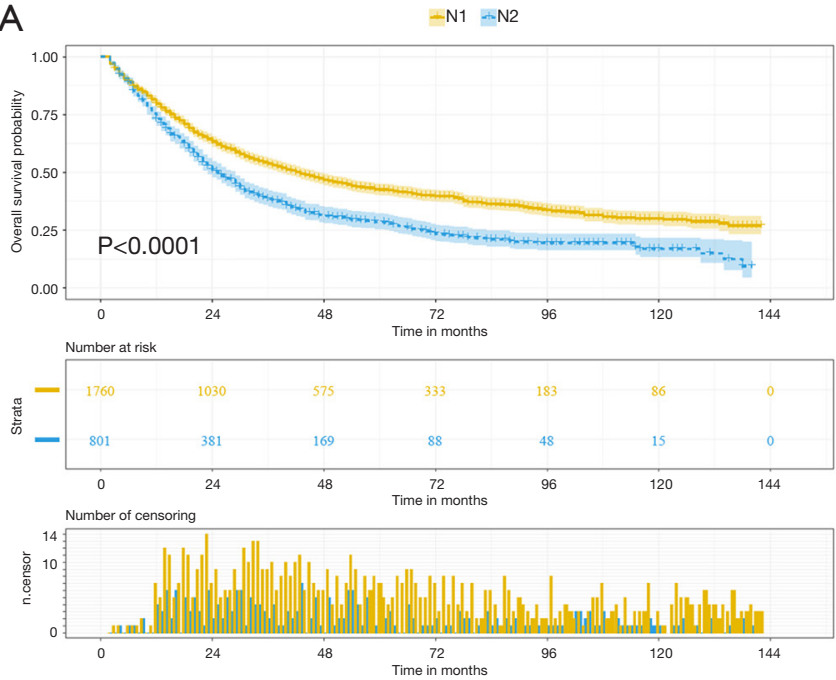

C
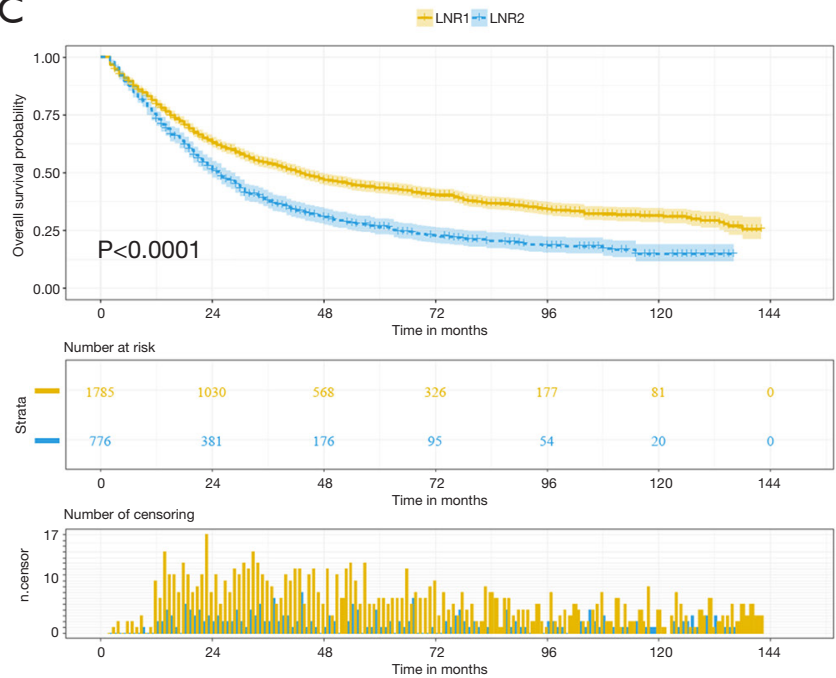

B
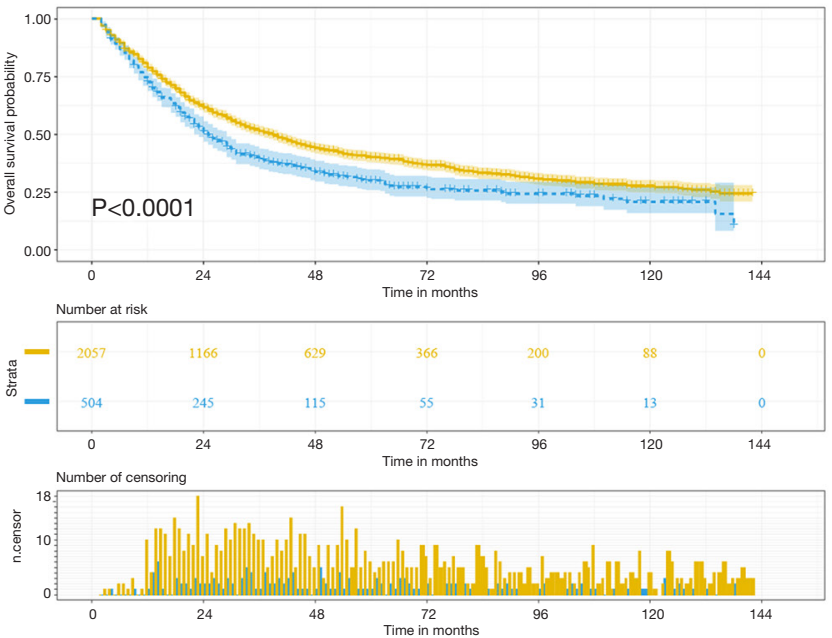

D $\quad$-LODDSTI=LODSS2
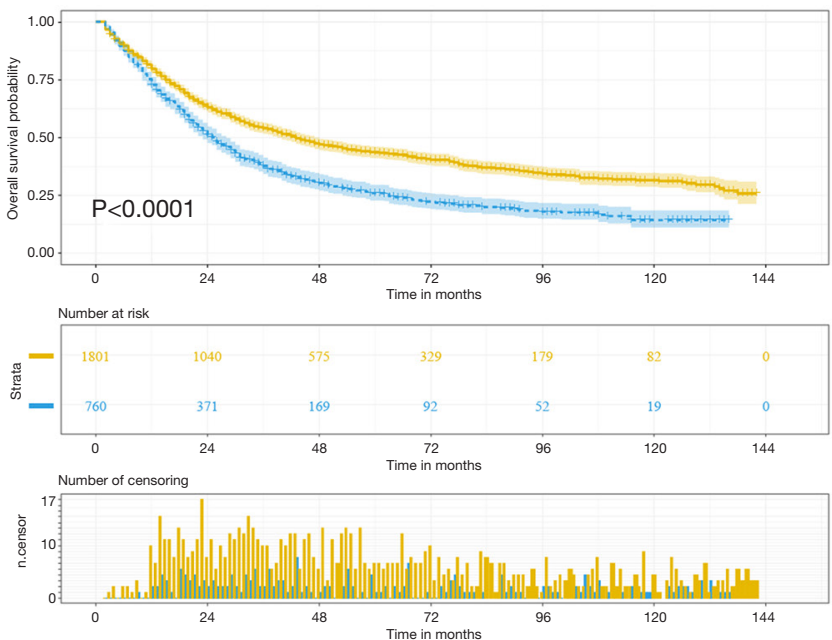

Figure 2 Kaplan-Meier estimates of overall survival for patients with N1/N2 stage SCC after surgery according to (A) N, (B) NPLN, (C) LNR, and (D) LODDS staging systems. SCC, squamous cell carcinoma; NPLN, number of positive lymph nodes; LNR, lymph node ratio; LODDS, log odds of positive lymph nodes.

Table 2 Univariable Cox regression analysis of potential prognostic predictors for cancer-specific survival and overall survival in patients with SCC

\begin{tabular}{|c|c|c|c|c|}
\hline \multirow{2}{*}{ Characteristic } & \multicolumn{2}{|c|}{ css } & \multicolumn{2}{|c|}{ os } \\
\hline & $\mathrm{HR}(95 \% \mathrm{Cl})$ & $P$ value & HR $(95 \% \mathrm{Cl})$ & $P$ value \\
\hline \multicolumn{5}{|c|}{ Year of diagnosis (year) } \\
\hline 2004-2005 & 1 & & 1 & \\
\hline 2006-2007 & $1.007(0.843,1.202)$ & 0.940 & $0.987(0.847,1.151)$ & 0.870 \\
\hline 2008-2009 & $1.049(0.882,1.248)$ & 0.588 & $1.024(0.879,1.192)$ & 0.762 \\
\hline $2010-2011$ & $0.873(0.730,1.043)$ & 0.135 & $0.825(0.704,0.967)$ & 0.017 \\
\hline
\end{tabular}

Table 2 (continued) 
Table 2 (continued)

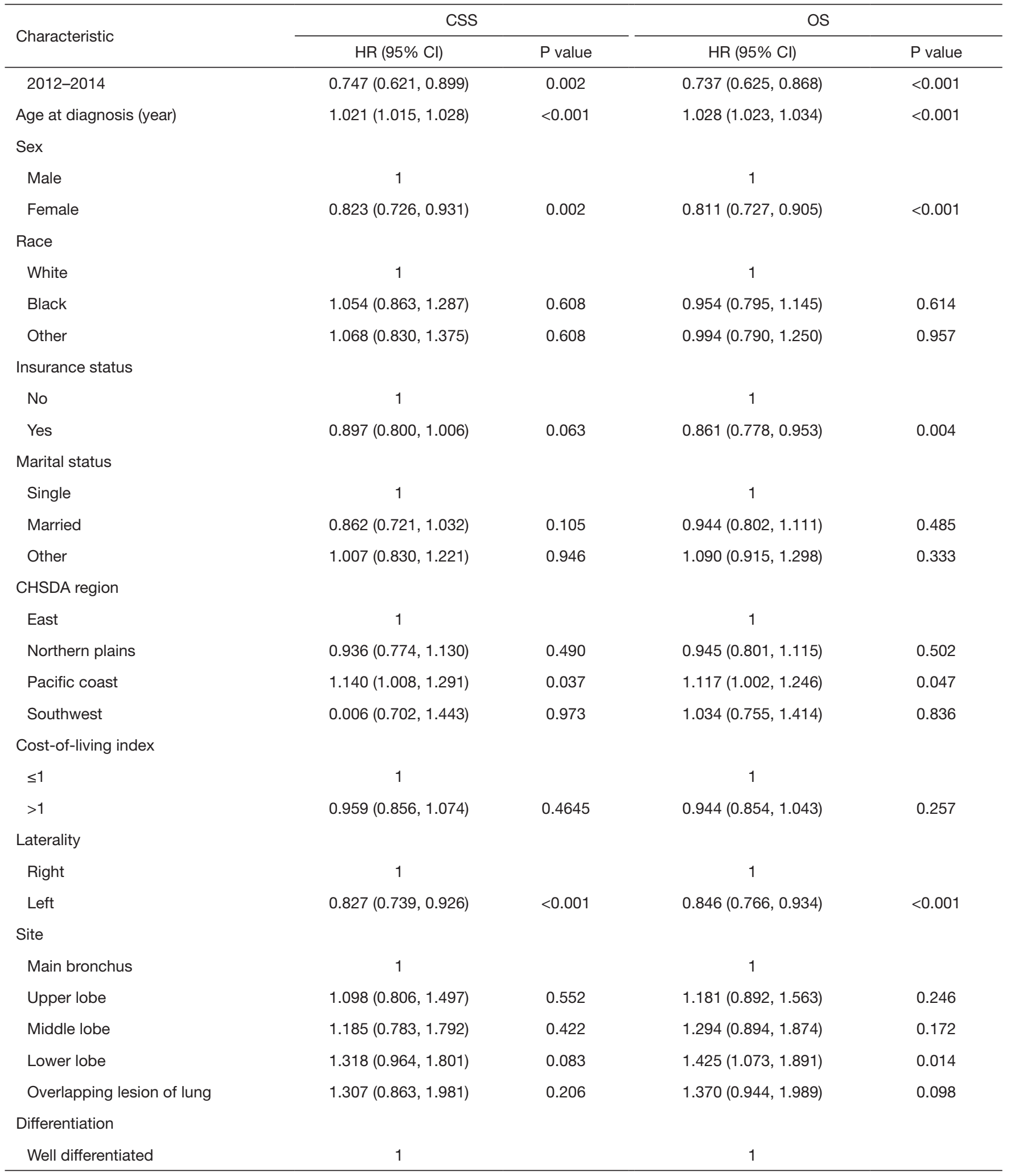

Table 2 (continued) 
Table 2 (continued)

\begin{tabular}{|c|c|c|c|c|}
\hline Characteristic & \multicolumn{2}{|c|}{ css } & \multicolumn{2}{|c|}{ os } \\
\hline Medium differentiated & $0.827(0.557,1.227)$ & 0.345 & $0.905(0.631,1.298)$ & 0.587 \\
\hline Poorly differentiated & $0.943(0.637,1.396)$ & 0.770 & $0.990(0.692,1.417)$ & 0.956 \\
\hline Undifferentiated & $1.356(0.751,2.451)$ & 0.313 & $1.192(0.685,2.074)$ & 0.535 \\
\hline $\mathrm{T} 2$ & $1.191(1.016,1.396)$ & 0.031 & $1.170(1.020,1.342)$ & 0.025 \\
\hline T3 & $1.614(1.296,2.010)$ & $<0.001$ & $1.455(1.197,1.769)$ & $<0.001$ \\
\hline $\mathrm{T} 4$ & $1.725(1.419,2.097)$ & $<0.001$ & $1.533(1.289,1.823)$ & $<0.001$ \\
\hline \multicolumn{5}{|l|}{ Type of surgery } \\
\hline Sublobectomy & 1 & & 1 & \\
\hline Lobectomy & $0.770(0.590,1.003)$ & 0.053 & $0.822(0.647,1.045)$ & 0.109 \\
\hline Pneumonectomy & $1.179(0.861,1.616)$ & 0.304 & $1.121(0.841,1.495)$ & 0.435 \\
\hline Unspecific & $0.807(0.610,1.068)$ & 0.134 & $0.839(0.652,1.079)$ & 0.171 \\
\hline \multicolumn{5}{|l|}{ Radiation } \\
\hline None/unknown & 1 & & 1 & \\
\hline Yes & $1.352(1.187,1.540)$ & $<0.001$ & $1.223(1.087,1.376)$ & $<0.001$ \\
\hline \multicolumn{5}{|l|}{ NDLN } \\
\hline NDLN2 [11-82] & $0.797(0.712,0.892)$ & $<0.001$ & $0.793(0.718,0.876)$ & $<0.001$ \\
\hline \multicolumn{5}{|l|}{ NPLN } \\
\hline NPLN1 [1-3] & 1 & & 1 & \\
\hline NPLN2 [4-33] & $1.421(1.244,1.624)$ & $<0.001$ & $1.318(1.169,1.487)$ & $<0.001$ \\
\hline \multicolumn{5}{|l|}{ LNR } \\
\hline LNR1 (0.01-0.28) & 1 & & 1 & \\
\hline LNR2 (0.29-1.00) & $1.636(1.457,1.837)$ & $<0.001$ & $1.518(1.369,1.684)$ & $<0.001$ \\
\hline \multicolumn{5}{|l|}{ LODDS } \\
\hline LODDS1 ( -1.70 to -0.37$)$ & 1 & & 1 & \\
\hline LODDS2 $(-0.36$ to 1.40$)$ & $1.654(1.473,1.858)$ & $<0.001$ & $1.540(1.388,1.708)$ & $<0.001$ \\
\hline
\end{tabular}

Note: data were presented as hazard ratio (HR) and a 95\% confidence interval (Cl). SCC, squamous cell carcinoma; CSS, cancer-specific survival; OS, overall survival; HR, Hazard ratio; Cl, confidence interval; CHSDA, contract health service delivery areas; NDLN, number of dissected lymph nodes; NPLN, number of positive lymph nodes; LNR, lymph node ratio; LODDS, log odds of positive lymph nodes. 
Table 3 Predictive performance comparison among the different nodal staging systems

\begin{tabular}{|c|c|c|c|c|}
\hline Study cohort & Linear trend $\chi^{2}$ score & AIC & LR test & C-index \\
\hline Model $1(\mathrm{~N})$ & 245.92 & $17,581.43$ & 202.35 & 0.6313 \\
\hline Model 2 (NPLN) & 232.45 & $17,551.48$ & 228.30 & 0.6392 \\
\hline Model 3 (LNR) & 248.62 & $17,537.13$ & 241.67 & 0.6484 \\
\hline Model 5 (N + NPLN + LODDS) & 300.03 & $17,494.31$ & 289.53 & 0.6619 \\
\hline \multicolumn{5}{|l|}{ Overall survival } \\
\hline Model $1(\mathrm{~N})$ & 358.23 & $22,401.33$ & 325.69 & 0.6404 \\
\hline Model 2 (NPLN) & 349.56 & $22,374.70$ & 348.32 & 0.6477 \\
\hline Model 5 (N + NPLN + LODDS) & 410.91 & $22,323.86$ & 403.51 & 0.6659 \\
\hline
\end{tabular}

AIC, Akaike information criterion; LR, likelihood ratio; C-index, Harrell concordance index; NPLN, number of positive lymph nodes; LNR, lymph node ratio; LODDS, log odds of positive lymph nodes.

$\mathrm{HR}=1.310 ; 95 \% \mathrm{CI}, 1.150-1.492)$ were identified to be independent prognostic factors in the Cox regression model (Tables 56,57 ). However, after adjusting for the N, NPLN, LODDS, and other variables, LNR didn't remain statistical significance anymore in Model 5, and had no effect on CSS and OS.

\section{Comparison of prognostic performance among $N, N P L N$, LNR, and LODDS}

The performance of each Cox regression model was assessed and summarized in Table 3. In Model 1-4, LODDS (Model 4) showed higher linear trend $\chi^{2}$ score (CSS: 250.46; OS: 364.08), lower AIC (CSS: 17,535.52; OS: 22,350.81), higher LR test (CSS: 242.95; OS: 358.82), and higher C-index (CSS: 0.6526; OS: 0.6617) compared to the N, NPLN, and LNR (Model 1-3). Therefore, it revealed that the LODDS might be superior to the N, NPLN, and LNR for predicting CSS and OS in patients with SCC. In Model 5, LNR was of no significance, which suggests that LODDS system might be a more reliable ratio-based LN method than LNR for nodal classification. Moreover, Model 5 showed the highest linear trend $\chi^{2}$ score (CSS: 300.03; OS: 410.91), lowest AIC (CSS: 17,494.31; OS: 22,323.86), highest LR test (CSS: 289.53; OS: 403.51), and highest C-index (CSS: 0.6619; OS: 0.6659), which demonstrated that the combination of the N, NPLN, and LODDS performed better than any staging system alone for predicting prognosis.

\section{Correlation between LODDS and N, NPLN, and LNR}

To illustrate the reason for the superiority of LODDS over the other systems, scatter plots were used to visualize the relationship between LODDS and the other three staging systems. LODDS was positively correlated with the N, NPLN and LNR ( $\mathrm{r}=0.266,0.446$, and 0.952 , respectively; $\mathrm{P}<0.001$ for all three variables) (Figure 3). Generally, the LODDS value increased with the increasing LNR value, while their correlation was not completely linear. When LNR was $\leq 0.1$ or $\geq 0.9$, the curves of LNR increased at a slower rate as compared to LODDS, especially when the value of LNR was proximity to 0 or 1 , indicating that LODDS system showed superiority for distinguishing heterogeneity. In addition, when NPLN was $\leq 10$, the LODDS system still kept heterogeneous, and could distinguish different survival outcomes for patients with the same NPLN.

\section{Sensitivity analysis and subgroup analysis}

Subgroup analysis on the basis of N, NPLN, and LNR were 

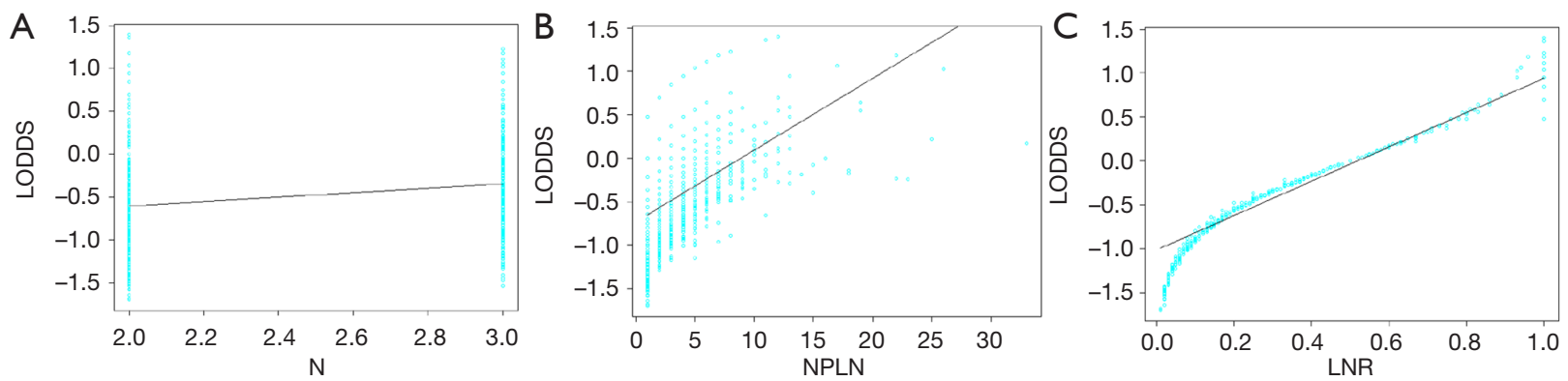

Figure 3 Scatter plots of the relationship between LODDS versus (A) N, (B) NPLN and (C) LNR in patients with N1/N2 stage SCC after surgery. SCC, squamous cell carcinoma; NPLN, number of positive lymph nodes; LNR, lymph node ratio; LODDS, log odds of positive lymph nodes.

\begin{tabular}{|c|c|c|c|c|c|c|}
\hline Subgroup & No. of Patients & LODDS1 & LODDS2 & & $\mathrm{HR}(95 \% \mathrm{Cl})$ & $P$ value \\
\hline \multicolumn{7}{|c|}{ Cancer specfic survival } \\
\hline \multicolumn{7}{|c|}{$\mathrm{N}$ stage } \\
\hline $\mathrm{N} 1$ & 1760 & 1368 & 392 & - & $1.569(1.341,1.837)$ & $<0.001$ \\
\hline N2 & 801 & 433 & 368 & $\rightarrow-$ & $1.448(1.206,1.739)$ & $<0.001$ \\
\hline \multicolumn{7}{|l|}{ NPLN } \\
\hline NPLN1 & 2057 & 1617 & 440 & $-\gamma$ & $1.574(1.364,1.817)$ & $<0.001$ \\
\hline NPLN2 & 504 & 184 & 320 & $\rightarrow-$ & $1.560(1.207,2.016)$ & $<0.001$ \\
\hline \multicolumn{7}{|l|}{ LNR } \\
\hline LNR1 & 1785 & 1785 & 0 & & NA & NA \\
\hline LNR2 & 776 & 16 & 760 & & $1.727(0.818,3.643)$ & 0.152 \\
\hline \multicolumn{7}{|c|}{ Overall survival } \\
\hline \multicolumn{7}{|c|}{$\mathrm{N}$ stage } \\
\hline N1 & 1760 & 1368 & 392 & $-\infty$ & $1.453(1.265,1.669)$ & $<0.001$ \\
\hline N2 & 801 & 433 & 368 & 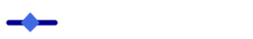 & $1.422(1.203,1.680)$ & $<0.001$ \\
\hline \multicolumn{7}{|l|}{ NPLN } \\
\hline NPLN1 & 2057 & 1617 & 440 & $-\infty$ & $1.474(1.298,1.674)$ & $<0.001$ \\
\hline NPLN2 & 504 & 184 & 320 & - & $1546(1.224,1.953)$ & $<0.001$ \\
\hline \multicolumn{7}{|l|}{ LNR } \\
\hline LNR1 & 1785 & 1785 & 0 & & NA & NA \\
\hline \multirow[t]{3}{*}{ LNR2 } & 776 & 16 & 760 & 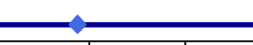 & $1.879(0.935,3.778)$ & 0.076 \\
\hline & & & $\begin{array}{lll}0.5 & 1\end{array}$ & 23 & & \\
\hline & \multicolumn{6}{|c|}{ <---LODDS2 Better--- ---LODDS1 Better---> } \\
\hline
\end{tabular}

Figure 4 The forest plot of HR (95\% CI) for cancer-specific survival and overall survival in subgroups of the N, NPLN, and LNR systems according to the LODDS stratification. HR, hazard ratio; CI, confidence interval; NPLN, number of positive lymph nodes; LNR, lymph node ratio; LODDS, log odds of positive lymph nodes; NA, not available.

performed to further validate the robustness of our findings. After stratifying by N, NPLN, and LNR, we analyzed the CSS and OS between disparate LODDS stages. As shown in Figure 4, we observed that the patients with LODDS 2 had significantly deteriorative CSS and OS compared to those with LODDS 1 in almost all subgroups (exception for LNR), indicating LODDS had excellent discriminatory power in stratifying prognosis of distinct cohorts.

\section{Discussion}

An exact assessment of the $\mathrm{LN}$ status plays a crucial role in the management of patients with NSCLC, including the selection of patients for adjuvant therapy and predicting patient prognosis. LODDS, a new nodal staging system, has showed its superiority for predicting prognosis in other cancers $(24,28,29,31)$. However, the prognostic value of LODDS for patients with SCC is unclear. In this study, we 
found that LODDS was an independent prognostic factor for predicting long-term CSS and OS among N1/N2 stage SCC patients after undergoing the surgery. In addition, LODDS showed better prognostic performance than the $\mathrm{N}$, NPLN, and LNR staging systems.

Nodal status is one of the most significant prognostic indictors for NSCLC. The current AJCC N staging system based on the anatomic location in the positive LNs of the pulmonary hila (N1) and mediastinum (N2) is widely used. Compared to LN count, the anatomical definition of LN station is more complicated, which thus might potentially cause inconsistent interpretation and misclassification of stage $(42,43)$. Additionally, the accuracy of the $\mathrm{N}$ system is significantly influenced by the insufficient NDLN, which might lead to stage migration in nodal classification (37). The NPLN system not only depends on the number of LNs sampled or dissected to begin with in the surgical intervention, but also depends on pathological procedures, which could affect the accuracy of positive LN counts. The LNR system combines both LN involved and the total number of LN harvested, which could theoretically overcome the limitations of number-based nodal classification system. Several previous studies demonstrated that LNR was a promising prognostic factor in patients with node-positive NSCLC, and was superior to the N and number-based nodal classification $(19,38,44)$. However, even if the patients have different numbers of metastatic LNs and total number of dissected LNs, they might get the same LNR. For example, it seems obvious that the prognosis for patients with 1 positive LN out of 1 dissected LN would be better than that for patients with 10 positive LNs out of 10 dissected LNs, even though they both have the same LNR of 1 . Another major disadvantage of LNR is a tight correlation with the number of LNs dissected. Therefore if there is a low number of LNs harvested especially in video assisted thoracic surgery (VATS), the efficacy of LNR is dubious (45). Moreover, LNR system will not distinguish the difference in survival for patients with node-negative disease.

Among these four staging systems, LODDS is the only variable that encompasses the numbers of dissected, positive, and negative LNs. LODDS have been identified as an independent prognostic factor, and showed better prognostic predictive power than other $\mathrm{LN}$ assessment classifications in breast, gastric, gastric adenocarcinoma, pancreatic cancer, colorectal cancer, esophageal squamous cell carcinoma (ESCC), and oral squamous cell carcinomas (OSCC) (28-31,37,46-48). Recently, there were several studies attempting to explore the prognostic role of LODDS for NSCLC $(16,25,26)$. Similar to our results, a single center study (26) limited their cohort in patients with lung AC alone, and found that LODDS had the highest ability to allow accurate prognostic stratification along with greatest model fit than LNR and NPLN. Deng et al. (25) demonstrated that LODDS was slightly superior to LNR for NSCLC with $<10$ resected LNs, and LNR was slightly superior to LODDS for NSCLC with $\geq 10$ resected LNs. Compared to our study, differences in the study time window, inclusion and exclusion criteria of the cohort (in which the majority of patients were diagnosed with $\mathrm{AC}$ ), and the covariates included in the cox regression models still existed. In another study, Lv et al. (16) found that LODDS was superior to N, and NPLN in stage I-IIIa NSCLC. However, in this single-institutional study, only 240 cases with SCC were included, which neither analyzed the correlation between LODDS and CSS, nor specified the numbers of negative or positive LNs dissected. Moreover, the prognostic performance of a combination of LODDS and other systems was not explored in above studies.

To our knowledge, the current study is the first to dedicatedly compare the prognostic performance of four different LN staging systems (N, NPLN, LNR, and LODDS) for predicting long-term CSS and OS in node-positive SCC, and preliminarily explore the possibility of a merger of the LODDS and other systems to strengthen the current AJCC TNM classification. In the multivariate Cox regression analysis (Model 1-4), patients with more advanced N stage, more NPLN, higher LNR, and higher LODDS were associated worse CSS and OS, which indicates that such patients might benefit from aggressive postoperative treatment or close follow-up. Moreover, Model 4 (LODDS) showed better performance in discriminatory power, monotonicity of gradients, homogeneity, and accuracy of prognostic prediction suggesting that the LODDS system might be superior to the other three staging systems when each of them was incorporated into Cox model separately. In Model 5, LODDS and other staging systems were combined together to provide more information, and showed the strongest prognostic effectiveness compared to Model 1-4, which demonstrated that LODDS could complement the widely used AJCC TNM classification and a combination of LODDS and other systems has the potential for becoming an better staging method to more precisely predicting survival. Moreover, a more accurate staging system could provide a tool for finer stratification 
to identify the most appropriate therapeutic strategies for specific subgroups of patients. A series of sensitivity analyses showed that LODDS had the ability to distinguish between patients with different $\mathrm{N}$ or NPLN subgroups into distinct prognostic subgroups. Scatter plots were used to visualize the relationship between LODDS and other staging systems. When LNR was 1 or less than 0.1 , and NPLN was less than 10, the value of LODDS was more heterogeneous, indicating that the LODDS system has the potential to discriminate survival outcomes among these patients. When comparing the prognostic effectiveness of different nodal staging systems, it is necessary to consider how many LNs should be dissected for adequate assessment of LN status and maintenance of the optimal quality of surgical intervention. However, the optimal number of LNs to be dissected has not been established with a range from 4 to 20 (49-53). In this current study, the optimal cut-off point of NDLN (NDLN1 $\leq 10$; NDLN2 $>10$ ) was calculated by X-tile analysis, and NDLN was found an independent prognostic indictor in Model 1, 2 and 5. An extensive LN dissection could facilitate the identification of stage migration, which may contribute to the proper delivery of adjuvant treatment. Furthermore, the number of LNs dissected serves as a quality measure of surgery, and adherence to lung cancer surgical quality standard is associated with better survival (49).

Several strengths and limitations of our study should be noted. The SEER program collects data on cancer patients from 18 registries throughout the United States, resulting in a high level of generalizability. Thus, our findings were based on a comprehensive setting and had strong external validity with a relatively large sample size in the analysis of node-positive SCC. Moreover, an adequate duration of follow-up time and relatively complete survival data provided sufficient power to assess prognostic performance of each staging system. Each Cox regression model was assessed by monotonicity, homogeneity, and distinctiveness, which made the evaluation of LN staging system more comprehensive. $\mathrm{X}$-tile is a bio-informatics statistical tool for biomarker assessment and outcome-based cut-point optimization, which is helpful to overcome the limitations of previous system grouping by using medians or quartiles as cut-off points. However, our study bears several limitations that we must acknowledge. The SEER database is the lack of detailed data, such as smoking history, imaging techniques used before surgery, preoperative chemotherapy, type of resection (R0, R1 or R2), use of systemic therapies, and postoperative comorbidities. In the SEER database, the AJCC stage derived from the collaborative stage (CS) system, represents a mixture of clinical and pathologic stage (https://seer.cancer.gov/tools/collabstaging/). Considering the discrepancies between these two staging systems, there is a need for further subgroup analysis regarding sole clinical stage or pathologic stage. In addition, information about recurrence is not available, and progression-free survival cannot be calculated. However, this current study showed comparable CSS rates, and CSS can be assumed to be an indirect extension of progression-free survival. In our study, categorization of continuous variables bears the risk of loss of information and is sometimes highly debated.

\section{Conclusions}

To summarize, this study confirmed the prognostic value of LODDS for predicting long-term CSS and OS in patients with node-positive lung SCC after undergoing surgical intervention. Additionally, LODDS system might have superior prognostic effectiveness over the N, NPLN, and LNR staging systems. Moreover, incorporation of LODDS into the AJCC $\mathrm{N}$ classification should be considered to more precisely predict survival, guide the choice of surgical procedure, and help tailor individualized postoperative treatment.

\section{Acknowledgments}

Funding: This work was supported by Military Medical Science and Technology Youth Cultivation Plan (17QNP013), Shanghai Municipal Commission of Science and Technology (17ZR1439100), Shanghai Shenkang Medicine Developing Project (SHDC12014107), and Shanghai Science and Technology Committee Medicine Leading Project (15411960100).

\section{Footnote}

Reporting Checklist: The authors have completed the TRIPOD reporting checklist. Available at http://dx.doi. org/10.21037/tlcr-20-193

Conflicts of Interest: All authors have completed the ICMJE uniform disclosure form (available at http://dx.doi. org/10.21037/tlcr-20-193). The authors have no conflicts of interest to declare.

Ethical Statement: The authors are accountable for all 
aspects of the work in ensuring that questions related to the accuracy or integrity of any part of the work are appropriately investigated and resolved. This study utilized the anonymous data available in the SEER database, and hence the requirement for informed consent was waived. This study was complied with the Declaration of Helsinki (as revised in 2013) and was approved by the Ethics Committee of Changzheng Hospital (No. ChiCTR-IOR-19005542).

Open Access Statement: This is an Open Access article distributed in accordance with the Creative Commons Attribution-NonCommercial-NoDerivs 4.0 International License (CC BY-NC-ND 4.0), which permits the noncommercial replication and distribution of the article with the strict proviso that no changes or edits are made and the original work is properly cited (including links to both the formal publication through the relevant DOI and the license). See: https://creativecommons.org/licenses/by-nc-nd/4.0/.

\section{References}

1. Fidler MM, Bray F, Soerjomataram I. The global cancer burden and human development: A review. Scand J Public Health 2018;46:27-36.

2. Torre LA, Bray F, Siegel RL, et al. Global cancer statistics, 2012. CA Cancer J Clin 2015;65:87-108.

3. Hirsch FR, Scagliotti GV, Mulshine JL, et al. Lung cancer: current therapies and new targeted treatments. Lancet 2017;389:299-311.

4. Malik PS, Sharma MC, Mohanti BK, et al. Clinicopathological profile of lung cancer at AIIMS: a changing paradigm in India. Asian Pac J Cancer Prev 2013;14:489-94.

5. Mao Y, Yang D, He J, et al. Epidemiology of Lung Cancer. Surg Oncol Clin N Am 2016;25:439-45.

6. Scagliotti GV, Parikh P, von Pawel J, et al. Phase III study comparing cisplatin plus gemcitabine with cisplatin plus pemetrexed in chemotherapy-naive patients with advanced-stage non-small-cell lung cancer. J Clin Oncol 2008;26:3543-51.

7. Sculier JP. Management of resectable non-small cell lung cancer. Guidelines of clinical practice made by the European Lung Cancer Working Party. Rev Med Brux 2014;35:134-9.

8. Kodera Y, Yamamura Y, Shimizu Y, et al. The number of metastatic lymph nodes: a promising prognostic determinant for gastric carcinoma in the latest edition of the TNM classification. J Am Coll Surg 1998;187:597-603.

9. Sinn HP, Helmchen B, Wittekind CH. TNM classification of breast cancer: changes and comments on the 7th edition. Pathologe 2010;31:361-6.

10. Gallegos-Hernández JF, Hernandez-Hernandez DM, Flores-Diaz R, et al. The number of sentinel nodes identified as prognostic factor in oral epidermoid cancer. Oral Oncol 2005;41:947-52.

11. Yang L, Xiong Z, Xie Q, et al. Prognostic value of total number of lymph nodes retrieved differs between leftsided colon cancer and right-sided colon cancer in stage III patients with colon cancer. BMC Cancer 2018;18:558.

12. Chu X, Yang ZF. Impact on survival of the number of lymph nodes resected in patients with lymph nodenegative gastric cancer. World J Surg Oncol 2015;13:192.

13. Goldstraw P, Chansky K, Crowley J, et al. The IASLC Lung Cancer Staging Project: Proposals for Revision of the TNM Stage Groupings in the Forthcoming (Eighth) Edition of the TNM Classification for Lung Cancer. J Thorac Oncol 2016;11:39-51.

14. Rusch VW, Chansky K, Kindler HL, et al. The IASLC Mesothelioma Staging Project: Proposals for the M Descriptors and for Revision of the TNM Stage Groupings in the Forthcoming (Eighth) Edition of the TNM Classification for Mesothelioma. J Thorac Oncol 2016;11:2112-9.

15. Tsim S, O'Dowd CA, Milroy R, et al. Staging of nonsmall cell lung cancer (NSCLC): a review. Respir Med 2010;104:1767-74.

16. Lv P, Chen G, Zhang P. Log odds of positive lymph nodes are superior to other measures for evaluating the prognosis of non-small cell lung cancer. Thorac Cancer 2014;5:570-5.

17. Taylor MD, LaPar DJ, Thomas CJ, et al. Lymph node ratio predicts recurrence and survival after $\mathrm{R} 0$ resection for non-small cell lung cancer. Ann Thorac Surg 2013;96:1163-70.

18. Wei S, Asamura H, Kawachi R, et al. Which is the better prognostic factor for resected non-small cell lung cancer: the number of metastatic lymph nodes or the currently used nodal stage classification? J Thorac Oncol 2011;6:310-8.

19. Chiappetta M, Leuzzi G, Sperduti I, et al. Lymph-node ratio predicts survival among the different stages of nonsmall-cell lung cancer: a multicentre analysis dagger. Eur J Cardiothorac Surg 2019;55:405-12.

20. Bi G, Lu T, Yao G, et al. The Prognostic Value Of Lymph Node Ratio In Patients With N2 Stage Lung Squamous Cell Carcinoma: A Nomogram And Heat Map Approach. Cancer Manag Res 2019;11:9427-37. 
21. Tamura M, Matsumoto I, Saito D, et al. Lymph node ratio as a prognostic factor in patients with pathological N2 non-small cell lung cancer. World J Surg Oncol 2016;14:295.

22. Wisnivesky JP, Arciniega J, Mhango G, et al. Lymph node ratio as a prognostic factor in elderly patients with pathological N1 non-small cell lung cancer. Thorax 2011;66:287-93.

23. Saji H, Tsuboi M, Shimada Y, et al. A proposal for combination of total number and anatomical location of involved lymph nodes for nodal classification in non-small cell lung cancer. Chest 2013;143:1618-25.

24. Kwon J, Eom KY, Kim IA, et al. Prognostic Value of Log Odds of Positive Lymph Nodes after Radical Surgery Followed by Adjuvant Treatment in High-Risk Cervical Cancer. Cancer Res Treat 2016;48:632-40.

25. Deng W, Xu T, Wang Y, et al. Log odds of positive lymph nodes may predict survival benefit in patients with node-positive non-small cell lung cancer. Lung Cancer 2018;122:60-6.

26. Zhao Y, Li G, Zheng D, et al. The prognostic value of lymph node ratio and log odds of positive lymph nodes in patients with lung adenocarcinoma. J Thorac Cardiovasc Surg 2017;153:702-709.e1.

27. Dziedzic D, Piotr R, Langfort R, et al. Log odds of positive lymph nodes as a novel prognostic indicator in NSCLC staging. Surg Oncol 2017;26:80-5.

28. Fang HY, Yang H, He ZS, et al. Log odds of positive lymph nodes is superior to the number- and ratio-based lymph node classification systems for colorectal cancer patients undergoing curative (R0) resection. Mol Clin Oncol 2017;6:782-8.

29. Sun Z, Xu Y, Li de M, et al. Log odds of positive lymph nodes: a novel prognostic indicator superior to the numberbased and the ratio-based $\mathrm{N}$ category for gastric cancer patients with R0 resection. Cancer 2010;116:2571-80.

30. La Torre M, Nigri G, Petrucciani N, et al. Prognostic assessment of different lymph node staging methods for pancreatic cancer with $\mathrm{R} 0$ resection: $\mathrm{pN}$ staging, lymph node ratio, log odds of positive lymph nodes. Pancreatology 2014;14:289-94.

31. Arslan NC, Sokmen S, Canda AE, et al. The prognostic impact of the log odds of positive lymph nodes in colon cancer. Colorectal Dis 2014;16:O386-92.

32. Cronin KA, Ries LA, Edwards BK. The Surveillance, Epidemiology, and End Results (SEER) Program of the National Cancer Institute. Cancer 2014;120 Suppl 23:3755-7.
33. Detterbeck FC. The eighth edition TNM stage classification for lung cancer: What does it mean on main street? J Thorac Cardiovasc Surg 2018;155:356-9.

34. Wang J, Hassett JM, Dayton MT, et al. The prognostic superiority of log odds of positive lymph nodes in stage III colon cancer. J Gastrointest Surg 2008;12:1790-6.

35. Camp RL, Dolled-Filhart M, Rimm DL. X-tile: a new bio-informatics tool for biomarker assessment and outcome-based cut-point optimization. Clin Cancer Res 2004;10:7252-9.

36. Huang B, Chen C, Ni M, et al. Log odds of positive lymph nodes is a superior prognostic indicator in stage III rectal cancer patients: A retrospective analysis of 17,632 patients in the SEER database. Int J Surg 2016;32:24-30.

37. Yang $M$, Zhang $H, M a Z$, et al. Log odds of positive lymph nodes is a novel prognostic indicator for advanced ESCC after surgical resection. J Thorac Dis 2017;9:1182-9.

38. Ding X, Hui Z, Dai H, et al. A Proposal for Combination of Lymph Node Ratio and Anatomic Location of Involved Lymph Nodes for Nodal Classification in Non-Small Cell Lung Cancer. J Thorac Oncol 2016;11:1565-73.

39. Deng J, Liang H, Wang D, et al. Enhancement the prediction of postoperative survival in gastric cancer by combining the negative lymph node count with ratio between positive and examined lymph nodes. Ann Surg Oncol 2010;17:1043-51.

40. Zhao C, Luo Q, Xia X, et al. Risk score to predict mortality in continuous ambulatory peritoneal dialysis patients. Eur J Clin Invest 2014;44:1095-103.

41. Harrell FE Jr, Lee KL, Mark DB. Multivariable prognostic models: issues in developing models, evaluating assumptions and adequacy, and measuring and reducing errors. Stat Med 1996;15:361-87.

42. El-Sherief AH, Lau CT, Obuchowski NA, et al. CrossDisciplinary Analysis of Lymph Node Classification in Lung Cancer on CT Scanning. Chest 2017;151:776-85.

43. Ramirez RA, Wang CG, Miller LE, et al. Incomplete intrapulmonary lymph node retrieval after routine pathologic examination of resected lung cancer. J Clin Oncol 2012;30:2823-8.

44. Han H, Zhao Y, Gao Z, et al. A prognostic score system with lymph node ratio in stage IIIA-N2 NSCLC patients after surgery and adjuvant chemotherapy. J Cancer Res Clin Oncol 2019;145:2115-22.

45. Merritt RE, Hoang CD, Shrager JB. Lymph node evaluation achieved by open lobectomy compared with thoracoscopic lobectomy for N0 lung cancer. Ann Thorac Surg 2013;96:1171-7. 
46. Bao X, Chen F, Qiu Y, et al. Log odds of positive lymph nodes is not superior to the number of positive lymph nodes in predicting overall survival in patients with oral squamous cell carcinomas. J Oral Maxillofac Surg 2020;78:305-12.

47. Agalar C, Aysal A, Unek T, et al. The role of log odds of positive lymph nodes in predicting the survival after resection for ampullary adenocarcinoma. Pathol Oncol Res 2020;26:467-73.

48. Ramacciato G, Nigri G, Petrucciani N, et al. Prognostic role of nodal ratio, LODDS, $\mathrm{pN}$ in patients with pancreatic cancer with venous involvement. BMC Surg 2017;17:109.

49. Dai J, Liu M, Yang Y, et al. Optimal Lymph Node Examination and Adjuvant Chemotherapy for Stage I Lung Cancer. J Thorac Oncol 2019;14:1277-85.

Cite this article as: Yu Y, Zhang P, Yao R, Wang J, Wang P, Xue X, Xiao J, Wang Z. Prognostic value of $\log$ odds of positive lymph nodes in node-positive lung squamous cell carcinoma patients after surgery: a SEER population-based study. Transl Lung Cancer Res 2020;9(4):1285-1301. doi: 10.21037/tlcr-20-193
50. Osarogiagbon RU, Ogbata O, Yu X. Number of lymph nodes associated with maximal reduction of long-term mortality risk in pathologic node-negative non-small cell lung cancer. Ann Thorac Surg 2014;97:385-93.

51. Becker DJ, Levy BP, Gold HT, et al. Influence of Extent of Lymph Node Evaluation on Survival for Pathologically Lymph Node Negative Non-Small Cell Lung Cancer. Am J Clin Oncol 2018;41:820-5.

52. Cao J, Xu J, He Z, et al. Prognostic impact of lymphadenectomy on outcomes of sublobar resection for stage IA non-small cell lung cancer $</=2 \mathrm{~cm}$. J Thorac Cardiovasc Surg 2018;156:796-805.e4.

53. Gulack BC, Yang CF, Speicher PJ, et al. The impact of tumor size on the association of the extent of lymph node resection and survival in clinical stage I non-small cell lung cancer. Lung Cancer 2015;90:554-60. 


\section{Supplementary}

Table S1 Number of observations after each selection procedure

\begin{tabular}{|c|c|c|c|}
\hline Step & Criteria & $\begin{array}{l}\text { Number } \\
\text { excluded }\end{array}$ & $\begin{array}{l}\text { Number of remaining } \\
\text { observations }\end{array}$ \\
\hline 1 & Patients with lung SCC between 2004 and 2014 & - & 109,348 \\
\hline 3 & Exclude if no histologically confirmation of invasive cancer & 13,427 & 94,982 \\
\hline 4 & Exclude if receiving a diagnosis while in a nursing home or hospice & 174 & 94,808 \\
\hline 6 & Include if aged $\geq 18$ years & 4 & 63,817 \\
\hline 7 & $\begin{array}{l}\text { Exclude if undergoing pathological examination of resected specimens or invasive } \\
\text { mediastinal staging before surgery }\end{array}$ & 55 & 63,762 \\
\hline 8 & Exclude if NO and N3 stage disease & 25,121 & 38,641 \\
\hline 11 & Include if at least one positive LN dissected & 2,656 & 4,112 \\
\hline 12 & Exclude if receiving preoperative radiotherapy & 437 & 3,675 \\
\hline 13 & Exclude if survival time of less than 1 month after surgery & 413 & 3,262 \\
\hline 14 & $\begin{array}{l}\text { Missing information about NDLN, NPLN, surgical approach, TNM staging as well as } \\
\text { survival outcomes }\end{array}$ & 701 & 2,561 \\
\hline
\end{tabular}

SCC, squamous cell carcinoma; LN, lymph node; NDLN, number of dissected lymph nodes; NPLN, number of positive lymph nodes; TNM, tumor node metastasis.

Table S2 1-, 3-, and 5-year lung cancer-specific survival rate of subgroups of different staging systems

\begin{tabular}{|c|c|c|c|c|}
\hline Study cohort & 1 -year survival rate $(\%)$ & 3-year survival rate (\%) & 5-year survival rate (\%) & $P$ value \\
\hline $\mathrm{N}$ & & & & $<0.001$ \\
\hline N1 & $84.3(82.5-86.0)$ & $61.2(58.8-63.7)$ & $52.0(49.3-54.7)$ & \\
\hline $\mathrm{N} 2$ & 76.5 (73.6-79.6) & $43.6(40.0-47.5)$ & 35.5 (31.9-39.6) & \\
\hline NPLN1 & $83.2(81.6-84.9)$ & $58.6(56.4-61.0)$ & $49.1(46.7-51.6)$ & \\
\hline NPLN2 & $76.0(72.3-79.9)$ & 43.8 (39.4-48.8) & $38.2(33.6-43.3)$ & \\
\hline LNR & & & & $<0.001$ \\
\hline LNR1 & 84.3 (82.6-86.1) & $61.2(58.8-63.7)$ & $52.7(50.1-55.4)$ & \\
\hline LODDS1 & $84.4(82.7-86.1)$ & $61.1(58.7-63.6)$ & $52.7(50.1-55.4)$ & \\
\hline LODDS2 & 75.7 (72.7-78.9) & $43.2(39.5-47.1)$ & 33.7 (30.0-37.7) & \\
\hline
\end{tabular}

Note: data were presented as survival rate (\%) with a 95\% confidence interval (Cl) in brackets; log-rank test was used to assess statistical differences between subgroups. NPLN, number of positive lymph nodes; LODDS, log odds of positive lymph nodes. 
Table S3 1-, 3-, and 5-year lung overall survival rate of subgroups of different staging systems

\begin{tabular}{|c|c|c|c|c|}
\hline Study cohort & 1-year survival rate $(\%)$ & 3-year survival rate (\%) & 5-year survival rate (\%) & $P$ value \\
\hline $\mathrm{N}$ & & & & $<0.001$ \\
\hline $\mathrm{N} 1$ & $79.6(77.7-81.5)$ & 53.7 (51.4-56.2) & $42.5(40.0-45.1)$ & \\
\hline N2 & 73.2 (70.2-76.3) & $38.6(35.2-42.3)$ & $28.4(25.1-32.1)$ & \\
\hline NPLN1 & $78.8(77.1-80.6)$ & $51.4(49.2-53.7)$ & $40.2(37.9-42.6)$ & \\
\hline NPLN2 & 72.6 (68.8-76.6) & $39.2(35.0-43.9)$ & $29.7(25.6-34.6)$ & \\
\hline LNR & & & & $<0.001$ \\
\hline LNR1 & $79.5(77.6-81.4)$ & $54.0(51.7-56.5)$ & $43.5(41.0-46.1)$ & \\
\hline LODDS1 & $79.6(77.7-81.4)$ & $54.0(51.6-56.4)$ & $43.6(41.1-46.2)$ & \\
\hline LODDS2 & 72.9 (69.8-76.1) & 37.6 (34.2-41.3) & 25.7 (22.5-29.4) & \\
\hline
\end{tabular}

Note: data were presented as survival rate (\%) with a 95\% confidence interval (Cl) in brackets; log-rank test was used to assess statistical differences between subgroups. NPLN, number of positive lymph nodes; LODDS, log odds of positive lymph nodes. 
Table S4 Multivariable Cox regression analysis (Model 1-4) of prognostic predictors for cancer-specific survival in patients with SCC

\begin{tabular}{|c|c|c|c|c|c|c|c|c|}
\hline \multirow{2}{*}{ Characteristic } & \multicolumn{2}{|l|}{ Model $1(\mathrm{~N})$} & \multicolumn{2}{|c|}{ Model 2 (NPLN) } & \multicolumn{2}{|c|}{ Model 3 (LNR) } & \multicolumn{2}{|c|}{ Model 4 (LODDS) } \\
\hline & $\mathrm{HR}(95 \% \mathrm{Cl})$ & $P$ value & $\mathrm{HR}(95 \% \mathrm{Cl})$ & $P$ value & HR (95\% Cl) & $P$ value & $\mathrm{HR}(95 \% \mathrm{Cl})$ & $P$ value \\
\hline Age group (year) & 1.019 (1.012-1.025) & $<0.001$ & 1.019 (1.013-1.026) & $<0.001$ & $1.018(1.012-1.025)$ & $<0.001$ & $1.018(1.012-1.025)$ & $<0.001$ \\
\hline \multicolumn{9}{|l|}{ Sex } \\
\hline Male & 1 & & 1 & & 1 & & 1 & \\
\hline Female & $0.782(0.690-0.886)$ & $<0.001$ & $0.795(0.702-0.901)$ & 0.0003 & $0.789(0.696-0.894)$ & $<0.001$ & $0.788(0.695-0.893)$ & $<0.001$ \\
\hline \multicolumn{9}{|l|}{ Insurance status } \\
\hline No & 1 & & 1 & & 1 & & 1 & \\
\hline Yes & $0.869(0.773-0.976)$ & 0.018 & $0.883(0.786-0.993)$ & 0.0381 & $0.892(0.793-1.003)$ & 0.056 & $0.892(0.793-1.003)$ & 0.049 \\
\hline \multicolumn{9}{|l|}{ Laterality } \\
\hline Right & 1 & & 1 & & 1 & & 1 & \\
\hline Left & $0.847(0.757-0.949)$ & 0.004 & $0.822(0.734-0.920)$ & 0.0006 & $0.827(0.739-0.926)$ & 0.001 & $0.830(0.742-0.929)$ & 0.001 \\
\hline \multicolumn{9}{|l|}{ T stage } \\
\hline $\mathrm{T} 1$ & 1 & & 1 & & 1 & & 1 & \\
\hline $\mathrm{T} 2$ & $1.251(1.066-1.468)$ & 0.006 & $1.244(1.060-1.460)$ & 0.0075 & $1.229(1.048-1.442)$ & 0.011 & $1.241(1.057-1.456)$ & 0.010 \\
\hline T3 & $1.878(1.504-2.345)$ & $<0.001$ & 1.757 (1.407-2.194) & $<0.001$ & $1.783(1.430-2.223)$ & $<0.001$ & $1.804(1.445-2.252)$ & $<0.001$ \\
\hline $\mathrm{T} 4$ & $1.890(1.550-2.305)$ & $<0.001$ & $1.838(1.508-2.241)$ & $<0.001$ & $1.811(1.487-2.207)$ & $<0.001$ & $1.828(1.500-2.229)$ & $<0.001$ \\
\hline \multicolumn{9}{|l|}{ Radiation } \\
\hline None/unknown & 1 & & 1 & & 1 & & 1 & \\
\hline Yes & 1.259 (1.094-1.450) & 0.001 & 1.373 (1.197-1.574) & $<0.001$ & $1.343(1.171-1.542)$ & $<0.001$ & $1.341(1.169-1.539)$ & $<0.001$ \\
\hline \multicolumn{9}{|l|}{ Chemotherapy } \\
\hline None/unknown & 1 & & 1 & & 1 & & 1 & \\
\hline Yes & $0.6722(0.5952-0.7591)$ & $<0.001$ & $0.687(0.609-0.775)$ & $<0.001$ & $0.673(0.596-0.759)$ & $<0.001$ & $0.676(0.599-0.763)$ & $<0.001$ \\
\hline \multicolumn{9}{|l|}{ NDLN } \\
\hline NDLN1 [1-10] & 1 & & 1 & & & & & \\
\hline NDLN2 [11-82] & $0.816(0.728-0.916)$ & $<0.001$ & $0.733(0.650-0.825)$ & $<0.001$ & & & & \\
\hline \multicolumn{9}{|l|}{$\mathrm{N}$ stage } \\
\hline N1 & 1 & & & & & & & \\
\hline N2 & $1.632(1.445-1.843)$ & $<0.001$ & & & & & & \\
\hline \multicolumn{9}{|l|}{ NPLN } \\
\hline NPLN1 [1-3] & & & 1 & & & & & \\
\hline NPLN2 [4-33] & & & $1.588(1.380-1.827)$ & $<0.001$ & & & & \\
\hline \multicolumn{9}{|l|}{ LNR } \\
\hline LNR1 (0.01-0.28) & & & & & 1 & & & \\
\hline LNR2 (0.29-1.00) & & & & & $1.648(1.464-1.856)$ & $<0.001$ & & \\
\hline \multicolumn{9}{|l|}{ LODDS } \\
\hline LODDS1 $(-1.70$ to -0.37$)$ & & & & & & & 1 & \\
\hline LODDS2 (-0.36 to 1.40$)$ & & & & & & & $1.661(1.475-1.870)$ & $<0.001$ \\
\hline
\end{tabular}

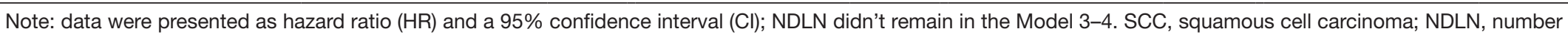
of dissected lymph nodes; NPLN, number of positive lymph nodes; LNR, lymph node ratio; LODDS, log odds of positive lymph nodes. 
Table S5 Multivariable Cox regression analysis (Model 1-4) of prognostic predictors for overall survival in patients with SCC

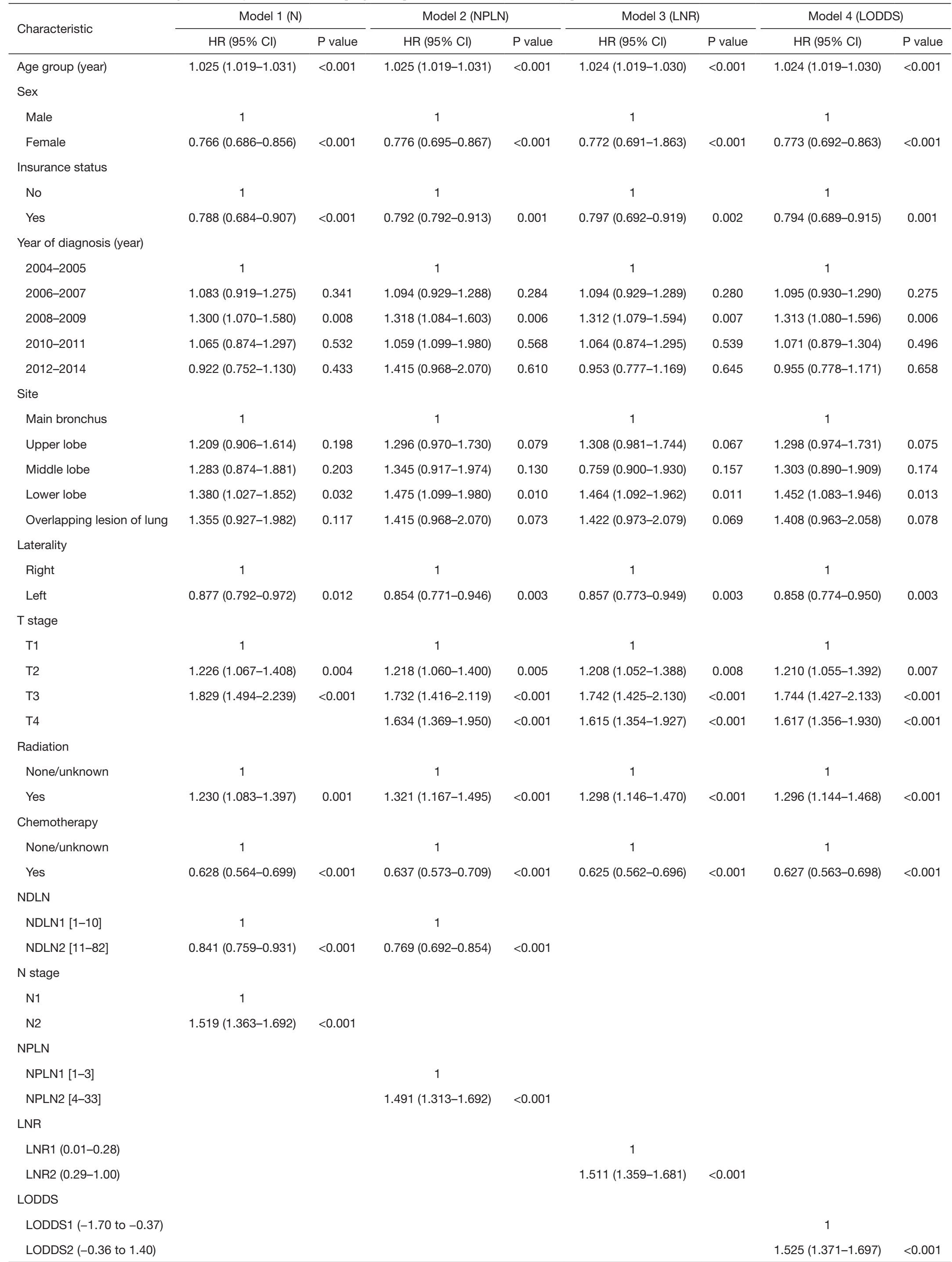

Note: data were presented as hazard ratio (HR) and a 95\% confidence interval (CI); NDLN didn't remain in the Model 3-4. SCC, squamous cell carcinoma; NDLN, number of dissected lymph nodes; NPLN, number of positive lymph nodes; LNR, lymph node ratio; LODDS, log odds of positive lymph nodes. 
Table S6 Multivariable Cox regression analysis (Model 5) of prognostic predictors for cancer-specific survival in patients with SCC

\begin{tabular}{|c|c|c|}
\hline \multirow{2}{*}{ Characteristic } & \multicolumn{2}{|l|}{ Model 5} \\
\hline & $\mathrm{HR}(95 \% \mathrm{Cl})$ & $P$ value \\
\hline Age at diagnosis (year) & $1.019(1.012-1.025)$ & $<0.001$ \\
\hline \multicolumn{3}{|l|}{ Sex } \\
\hline Male & 1 & \\
\hline Female & $0.779(0.688-0.883)$ & $<0.001$ \\
\hline \multicolumn{3}{|l|}{ Insurance status } \\
\hline No & 1 & \\
\hline Yes & $0.890(0.791-1.001)$ & 0.054 \\
\hline \multicolumn{3}{|l|}{ Laterality } \\
\hline Right & 1 & \\
\hline Left & $0.835(0.746-0.935)$ & 0.002 \\
\hline \multicolumn{3}{|l|}{ T stage } \\
\hline $\mathrm{T} 1$ & 1 & \\
\hline $\mathrm{T} 2$ & $1.233(1.051-1.447)$ & 0.010 \\
\hline T3 & $1.856(1.486-2.318)$ & $<0.001$ \\
\hline $\mathrm{T} 4$ & $1.891(1.551-2.306)$ & $<0.001$ \\
\hline \multicolumn{3}{|l|}{ Radiation } \\
\hline None/unknown & 1 & \\
\hline Yes & $1.202(1.043-1.386)$ & 0.011 \\
\hline \multicolumn{3}{|l|}{ Chemotherapy } \\
\hline None/unknown & 1 & \\
\hline Yes & $0.669(0.592-0.755)$ & $<0.001$ \\
\hline \multicolumn{3}{|l|}{ NDLN } \\
\hline NDLN1 [1-10] & 1 & \\
\hline NDLN2 [11-82] & $0.866(0.755-0.993)$ & 0.039 \\
\hline \multicolumn{3}{|l|}{$\mathrm{N}$ stage } \\
\hline $\mathrm{N} 1$ & 1 & \\
\hline N2 & $1.482(1.307-1.681)$ & $<0.001$ \\
\hline \multicolumn{3}{|l|}{ NPLN } \\
\hline NPLN1 [1-3] & 1 & \\
\hline NPLN2 [4-33] & $1.214(1.031-1.429)$ & 0.020 \\
\hline \multicolumn{3}{|l|}{ LODDS } \\
\hline LODDS1 $(-1.70$ to -0.37$)$ & 1 & \\
\hline LODDS2 (-0.36 to 1.40$)$ & $1.373(1.186-1.591)$ & $<0.001$ \\
\hline
\end{tabular}

Note: data were presented as hazard ratio (HR) and a $95 \%$ confidence interval (Cl); LNR didn't remain in Model 5. SCC, squamous cell carcinoma; HR, hazard ratio; $\mathrm{Cl}$, confidence interval; NDLN, number of dissected lymph nodes; NPLN, number of positive lymph nodes; LODDS, log odds of positive lymph nodes.
Table S7 Multivariable Cox regression analysis (Model 5) of prognostic predictors for overall survival in patients with SCC

\begin{tabular}{lcc}
\hline \multirow{2}{*}{ Characteristic } & \multicolumn{2}{c}{ Model 5 } \\
\cline { 2 - 3 } & HR $(95 \%$ Cl $)$ & P value \\
\hline Age group (year) & $1.025(1.019-1.031)$ & $<0.001$ \\
Sex & & \\
Male & 1 & \\
Female & $0.763(0.684-0.853)$ & $<0.001$
\end{tabular}

Insurance status

No

Yes

$0.848(0.764-0.941) \quad 0.002$

Year of diagnosis (year)

2004-2005

2006-2007

2008-2009

2010-2011

2012-2014

Site

Main bronchus

Upper lobe

1.266 (0.948-1.691)

0.111

Middle lobe

1.323 (0.901-1.944)

0.153

Lower lobe

1.428 (1.063-1.919)

0.018

Overlapping lesion of lung

$1.405(0.960-2.055)$

0.080

Laterality

Right

Left

1

$0.857(0.776-0.947) \quad 0.002$

T stage

T1

$\mathrm{T} 2$

$1.231(1.072-1.413) \quad 0.003$

$1.718(1.409-2.094) \quad<0.001$

T3

$1.700(1.425-2.027) \quad<0.001$

Radiation

None/unknown

Yes

1

$1.169(1.028-1.328) \quad 0.017$

Chemotherapy

None/unknown

Yes

1

$0.618(0.555-0.687)<0.001$

NDLN

NDLN1 [1-10]

NDLN2 [11-82]

1

$0.870(0.772-0.980) \quad 0.022$

$\mathrm{N}$ stage

N1

N2

1

$1.403(1.255-1.569) \quad<0.001$

NPLN

NPLN1 [1-3]

NPLN2 [4-33]

1

$1.193(1.030-1.381) \quad 0.019$

LODDS

LODDS1 $(-1.70$ to -0.37$)$

1

LODDS2 (-0.36 to 1.40) $1.310(1.150-1.492) \quad<0.001$

Note: data were presented as hazard ratio (HR) and a $95 \%$ confidence interval (Cl); LNR didn't remain in Model 5. SCC, squamous cell carcinoma; $\mathrm{HR}$, hazard ratio; $\mathrm{Cl}$, confidence interval; NDLN, number of dissected lymph nodes; NPLN, number of positive lymph nodes; LODDS, log odds of positive lymph nodes. 